\title{
The New World Mathematics Awards
}

\section{by the Editors}

Mathematics and mathematics education is of great significance in human pursuit of knowledge and truth. It is the foundation of science and technology. In 2007, Dr Henry Cheng (Managing Director of New World Development Company Limited) and Professor Shing-Tung Yau (Harvard University) initiated the New World Mathematics Awards (NWMA). The goal of this program is to encourage

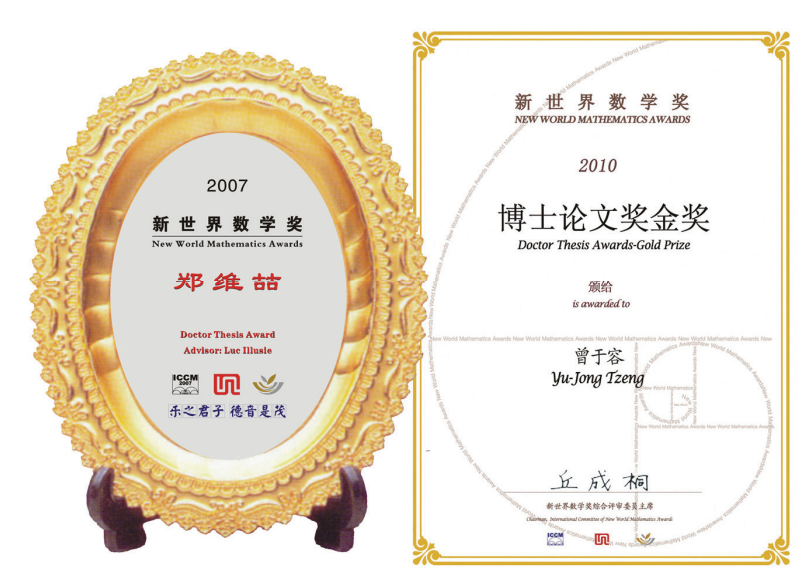

The New World Mathematics Doctor Thesis Gold Prize was awarded to Yu-Jong Tzeng in 2010

outstanding Chinese mathematics students worldwide in their pursuit of mathematical truth.

NWMA web page:

http://msc.tsinghua.edu.cn/nwma2013/

The NWMA is sponsored by the New World Development Company Limited and China Young Leaders Foundation (Chairman: Chi Kong Cheng), and Tsinghua University at Beijing. Chinese undergraduate, master and $\mathrm{PhD}$ mathematics students all over the world are encouraged to apply this award. The thesis topics may be pure or applied mathematics (including probability, statistics, biomathematics etc.).

\section{Application Process}

The candidate should submit the thesis, an application form, and one recommendation letter. The application form may be filled in online and the thesis be uploaded on the webpage of NWMA.

\section{Selection Process}

All submissions are subjected to a two-round review conducted by a scientific committee and then an international committee chaired by Professor Yau Shing-Tung. All members of these committees are world-renowned mathematicians.

\section{Qualification}

This program is open to all undergraduate, master and $\mathrm{PhD}$ mathematics Chinese students. The submitted thesis must be successfully defended and accepted by the students' college/university. Students have to show some evidence or document that the defense of thesis was finished.

The NWMA was announced in the ICCM 2007. From 2009 , it was announced yearly.

\section{Awardees of 2007 New World Mathematics Awards}

\section{Doctor Thesis Awards-Gold Prize}

Hao $\mathrm{Xu}$, Zhejiang University

Thesis title: Intersection numbers on moduli spaces of curves

Advisor: Professor Kefeng Liu

Current affiliation: Postdoc at Harvard Mathematics Department

Weizhe Zheng, Université Paris-Sud 11

Thesis title: Intégralité, rationalité et indépendance de $l$ en cohomologie l-adique sur les corps locaux

Advisor: Professor Luc Illusie

Current affiliation: Research Fellow, Morningside Center of Mathematics and Institute of Mathematics, Chinese Academy of Sciences, Beijing

\section{Doctor Thesis Awards-Silver Prize}

Xi Chen, Tsinghua University

Thesis title: On The Complexities of Two-Person Nash Equilibria

Advisor: Professor Bo Zhang 
Current affiliation: Assistant professor in the Computer Science Department of Columbia University

Jeng-Daw Yu, Harvard University

Thesis title: On ordinary K3 surfaces over Fp

Advisor: Professor Shing-Tung Yau

Current affiliation: Assistant Professor, Department

of Mathematics, National Taiwan University

Wanke Yin, Wuhan University

Thesis title: A Bishop surface with a vanishing Bishop invariant

Advisor: Professor Xiaojun Huang

Current affiliation: Associate Professor, Wuhan University

Ruonan Zhang, Stanford University

Thesis title: Change-point Detection and Sequence Alignment: Statistical Problems of Genomics

Advisor: Professor David Siegmund

Current affiliation: Assistant Professor of Statistics, Stanford University

\section{Master Thesis Awards-Gold Prize}

Nai-Chia Chen, National Tsinghua University, Hsinchu, Taiwan

Thesis title: On Symmetric Planar Central Configurations for the 5-Body Problem with 3 Collinear Masses

Advisor: Professor Kuo-Chang Chen

Current affiliation: Graduate Assistants, University of Minnesota

\section{Master Thesis Awards-Silver Prize}

Bin Dong, National University of Singapore

Thesis title: Pseudo-Splines, Wavelets and Framelets

Advisor: Professor Zuowei Shen

Current affiliation: Assistant Professor, Department of Mathematics, The University of Arizona

Rong Du, East China Normal University

Thesis title: Cubic Equations of Rational Triple Points Advisor: Professor Shengli Tan

Current affiliation: Associate Professor, Department of Mathematics, East China Normal University

Zhi Jiang, École Normale Supérieure

Thesis title: On the restriction of holomorphic forms

Advisor: Professor Claire Voisin

Current affiliation: Université Paris-Sud

Xia Li, Nanjing University

Thesis title: Plateau of $\alpha$-Function and c-minimal Homoclinic orbits

Advisor: Professor Chongqing Cheng

Po-Lam Yung, Chinese University of Hong Kong

Thesis title: Doubling properties of Self-Similar Measures

Advisor: Professor Ka-Sing Lau
Current affiliation: Hill Assistant Professor, Department of Mathematics, Rutgers University

\section{Bachelor Thesis Awards-Gold Prize}

Wei Deng, Zhejiang University

Thesis title: h-Cobordism Theorem

Advisor: Professor Wei Luo

Chin-Lung Fong, Chinese University of Hong Kong

Thesis title: Characterization of Entropy Vectors for Multi-User Information Theory

Advisor: Professor Ka-Sing Lau

Hongbin Sun, Peking University

Thesis title: Commensurability and Rational Commensurability

Advisor: Professor Shicheng Wang

Cheng-Chiang Tsai, National Taiwan University

Thesis title: On the behavior of Newton iteration

Advisor: Professor Chin-Lung Wang

Junjie Zhou, University of Science and Technology of China

Thesis title: Complex manifolds and Deformation

Advisor: Professor Sen $\mathrm{Hu}$

\section{Bachelor Thesis Awards-Silver Prize}

Xiaoshan Chen, South China Normal University

Thesis title: Solving the biharmonic equation on upper plane by the method of Fourier transformation Advisor: Professor Fahuai Yi

Yuke Huang, Tsinghua University

Thesis title: Fractal analysis of the market-the fractional Brownian motion model with drift

Advisor: Professor Hui Rao

Baiying Liu, Harbin Institute of Technology

Thesis title: Toric Varieties and Their Application

Advisor: Professor Sheng Chen

Binbin Lin, Zhejiang University

Thesis title: Image Restoration Based on Bayesian Analysis

Advisor: Professor Ligang Liu

Yun Yue, Zhejiang University

Thesis title: Deforming metrics with positive curvature by a fully nonlinear flow

Advisor: Professor Weimin Sheng

\section{Awardees of 2009 New World Mathematics Awards}

\section{Doctor Thesis Awards-Gold Prize}

Chen-Yu Chi, Harvard University

Thesis title: The pseudonorms and theorems of Torelli type for birational equivalence 
Advisor: Professor Shing-Tung Yau

Current affiliation: Assistant Professor, National Taiwan University

Yongquan $\mathrm{Hu}$, Université Paris-Sud 11

Thesis title: On p-adic and modulo p-local Langlands program

Advisor: Professor Christophe Breuil

Current affiliation: Matre de conférences, Université de Rennes 1

Jun Yin, Princeton University

Thesis title: Quantum many-body systems with short-range interactions

Advisor: Professor Robert Seiringer

Current affiliation: Assistant Professor, University of Wisconsin-Madison

\section{Doctor Thesis Awards-Silver Prizes}

Kwokwai Chan, The Chinese University of Hong Kong

Thesis title: Strominger-Yau-Zaslow transformations in mirror symmetry

Advisor: Professor Nai Chung (Conan) Leung

Current affiliation: Assistant Professor, The Chinese

University of Hong Kong

Kai-Wen Lan, Harvard University

Thesis title: Arithmetic compactifications of PEL-type Shimura varieties

Advisor: Professor Richard Taylor

Current affiliation: Assistant Professor, University of Minnesota

Wei-Dong Liu, Zhejiang University

Thesis title: Limit properties for a class of stationary processes

Advisor: Professor Zhengyan Lin

Current affiliation: Professor, Shanghai Jiao Tong University

Lok-Ming Lui, University of California at Los Angeles

Thesis title: Computational conformal geometry and its applications to human brain mapping

Advisor: Professor Tony Chan

Current affiliation: Assistant Professor, The Chinese

University of Hong Kong

Jilong Tong, University Paris-Sud Orsay

Thesis title: Applications d'Albanese pour les courbes et contractions and to diviseur theta et formes différentielles

Advisor: Professor Michel Raynaud

Current affiliation: Matre de conférences, Université de Bordeaux 1

\section{Master Thesis Awards-Gold Prize}

Sz-Sheng Wang, National Taiwan University

Thesis title: On extensions of multiply twisted pluri-canonical forms
Advisor: Professor Chin-Lung Wang

Current affiliation: National Taiwan University

\section{Master Thesis Awards-Silver Prize}

Yun Kuen Cheung, Hong Kong University of Science and Technology

Thesis title: Analysis of weighted digital sums by Mellin transform

Advisor: Professor Shiu-Yuen Cheng

Current affiliation: Graduate student, Courant Institute, New York University

Wen Deng, Université Pierre et Marie Curie

Thesis title: Spectral asymptotics for large skew-symmetric perturbations of the harmonic oscillator and generalizations

Advisor: Professor Nicolas Lerner

Current affiliation: Graduate student, Université Pierre et Marie Curie (Paris VI)

Sin Tsun (Edward) Fan, The Chinese University of Hong Kong

Thesis title: Open orbits and augmentations of Dynkin diagrams

Advisor: Professor Nai-Chung Leung

Current affiliation: Graduate student, California Institute of Technology

\section{Bachelor Thesis Awards-Gold Prize}

Ka Kit Lam, The Chinese University of Hong Kong Thesis title: Achieving capacity of network Advisor: Professor Babak Hassibi

Liang Zhao, Tsinghua University

Thesis title: Transcendence of hypergeometric functions for the function fields Advisor: Professor Jia-Yan Yao

\section{Bachelor Thesis Awards-Silver Prize}

Chao Li, Tsinghua University

Thesis title: Mathematical typesetting for computer algebra system

Advisor: Professor Hui Rao

Co-author: Wei Ruan, Long Zhang, Xiang Zhang

Dai Shi, Fudan University

Thesis title: Upper bounds on the number of eigenvalues of a steady-state Schrödinger equation

Advisor: Professor Zixiang Zhou

Chao Xu, Zhejiang University

Thesis title: Hyperbolic Kähler-Ricci flow

Advisor: Professor De-Xing Kong 


\section{Awardees of 2010 New World Mathematics Awards}

\section{Doctor Thesis Awards-Gold Prize}

Yu-Jong Tzeng, Stanford University

Thesis title: A proof of the Göttsche-Yau-Zaslow formula

Advisor: Professor Jun Li

Current affiliation: Simons Fellow, Harvard University

\section{Doctor Thesis Awards-Silver Prizes}

Rong Du, East China Normal University

Thesis title: Moduli space of bounded complete Reinhardt domains and complex Plateau problem Advisor: Professor Stephen Yau Current affiliation: Associate Professor, East China Normal University

Xiong Jin, Université Paris-Sud 11

Thesis title: Construction and multifractal analysis of random functions and their graphs

Advisor: Professor Julien Barral

Current affiliation: Research staff, University of St Andrews

Wanqiang Shen, Zhejiang University

Thesis title: Researches of theories and applications of changeable degree B-splines

Advisor: Professor Guozhao Wang

Current affiliation: Jiangnan University, Wuxi, China

Shenghao Sun, University of California, Berkeley

Thesis title: On l-adic cohomology of Artin stacks: L-functions, weights and the decomposition theorem Advisor: Professor Martin Olsson

Current affiliation: Lecturer, MSC,Tsinghua University

\section{Master Thesis Awards-Gold Prize}

Weihua Liu, Zhejiang University

Thesis title: On some problems of quantum measurement

Advisor: Professor Junde Wu

Current affiliation: Graduate student, UC Berkeley

\section{Master Thesis Awards-Silver Prize}

Ka Shing Ng, The Chinese University of Hong Kong

Thesis title: A survey on free probability

Advisor: Professor Chi-Wai Leung

Current affiliation: Graduate student, University of Waterloo

Yifei Zhong, National University of Singapore

Thesis title: Two free boundary problems in optimal investment

Advisor: Professor Min Dai

Current affiliation: Graduate student, Mathematical Institute, Oxford University
Bachelor Thesis Awards-Gold Prize

Heung Shan Theodore Hui, The Chinese University of Hong Kong

Thesis title I: On the additive and multiplicative properties of recurrence sequences

Advisor: Ms Yau-Man Sum and Mr Gesing Leung

Thesis title II: The upper bound of elliptic curves with quadratic twists

Advisor: Professor John Coates

Thesis title III: A computational approach on preperiodic points of rational polynomials

Advisor: Professor Kevin Hare and Professor Patrick Ingram.

\section{Bachelor Thesis Awards-Silver Prize}

Zhilan Wang, Tsinghua University

Thesis title: Arithmetic study of moduli space of algebraic curves of genus 0

Advisor: Professor Jian Zhou

Zhiyuan Xu, Zhejiang University

Thesis title: Rigidity theorems for compact minimal hypersurfaces in a sphere

Advisor: Professor Hongwei Xu

Xin Zhang, Zhejiang University

Thesis title: The eigenvalue problem of Schrödinger operator on minimal submanifolds of Euclidean space

Advisor: Professor Hongwei Xu

Xiaobo Zhuang, Tsinghua University

Thesis title: Arithmetic calculations of moduli spaces of representations of some quivers

Advisor: Professor Jian Zhou

\section{Awardees of 2011 New World Mathematics Awards}

\section{Doctor Thesis Awards-Gold Prize}

Shan Peng, Université Paris 7

Thesis title: Canonical bases and gradings associated with rational double affine

Hecke algebras

Advisor: Professor Eric Vasserot

Current affiliation: C L E Moore Instructor, MIT

Si Li, Harvard

Thesis title: Calabi-Yau Geometry and Higher Genus Mirror Symmetry

Advisor: Professor Shing-Tung Yau

Current affiliation: Assistant Professor, Boston University 
Doctor Thesis Awards-Silver Prizes

Po-Ning Chen, Harvard University

Thesis title: Quasi-local energy in General Relativity

Advisor: Professor Shing-Tung Yau

Current affiliation: Ritt Assistant Professor, Columbia

University

Yang Feng, Princeton University

Thesis title: High-dimensional Statistical Learning and Nonparametric Modeling

Advisor: Professor Jianqing Fan

Current affiliation: Assistant Professor, Columbia University

Xiangxiong Zhang, Brown University

Thesis title: Maximum-Principle-Satisfying and Positivity-Preserving High Order

Schemes for Conservation Laws

Advisor: Professor Chi-Wang Shu

Current affiliation: Postdoctoral Assosicate, MIT

Wenxuan Lu, University of Pennsylvania

Thesis title: Instanton Correction,Wall Crossing and Mirror Symmetry of Hitchin's Moduli Spaces

Advisor: Professor Shing-Tung Yau

Current affiliation: Lecturer, University of Pennsylvania

\section{Doctor Thesis Awards-Bronze Prizes}

Chung-Jun Tsai, Harvard University

Thesis title: Asymptotic spectral flow for Dirac operators of disjoint Dehn twists

Advisor: Professor Clifford Henry Taubes

Current affiliation: Postdoc, Harvard University

Shengmao Zhu, Zhejiang University

Thesis title: Moduli space, quantum invariant and topological string

Advisor: Professor Kefeng Liu

Current affiliation: Postdoc, Center of Mathematical

Sciences, Zhejiang University

Bachelor Thesis Awards-Gold Prize

Weichen $\mathrm{Gu}$, Tsinghua University

Thesis title: The Change of Dimension under Maps in Sobolev Spaces

Advisor: Professor Zhiying Wen

\section{Awardees of 2012 New World Mathematics Awards}

\section{Doctor Thesis Awards-Gold Prize}

Xiangyu Liang, University of Paris Sud 11

Thesis title: Ensembles et cônes minimaux dans les espaces euclidiens (minimal sets and cones in Euclidean spaces)
Advisor: Professor Guy David

Current affiliation: Postdoctoral research fellow, University of Warwick

Siu-Cheong Lau, The Chinese University of Hong Kong

Thesis title: SYZ Mirror Symmetry for Toric Calabi-Yau Manifolds

Advisor: Professor Naichung Conan Leung

Current affiliation: Benjamin Peirce lecturer, Harvard University

Zhiren Wang, Princeton University

Thesis title: On Higher Rank Commutative Actions by Toral Automorphisms

Advisor: Professor Elon Lindenstrauss

Current affiliation: Gibbs Assistant Professor, Yale University

\section{Doctor Thesis Awards-Silver Prizes}

Shangkun Weng, The Chinese University of Hong Kong Thesis title: On Multi-dimensional Steady Subsonic Flows Determined by Physical Boundary Conditions

Advisor: Professor Zhouping Xin

Current affiliation: Postdoc, Harvard University

Chun-Kit Lai, The Chinese University of Hong Kong

Thesis title: Spectral Analysis on Fractal Measures and Tiles

Advisor: Professor Ka-Sing Lau

Current affiliation: Postdoctoral Fellow, McMaster University

Wen Deng, Université Pierre et Marie Curie (Paris VI)

Thesis title: Etude du pseudo-spectre d'opérateurs non auto-adjoints liés à la mécanique des fluides

Advisor: Professor Nicolas Lerner

Current affiliation: Doctorant, Université Pierre et Marie Curie (Paris VI)

\section{Master Thesis Awards-Gold Prize}

Hao Wu, Université Paris-Sud

Thesis title: On the occupation times of Brownian excursions and Brownian loops

Advisor: Professor Wendelin Werner

Current affiliation: Université Paris-Sud

Tianwen Luo, The Chinese University of Hong Kong

Thesis title: Nonuniquess of Weak Solutions to the Compressible Euler Equations

Advisor: Professor Zhouping Xin

Current affiliation: The Chinese University of Hong Kong

Master Thesis Awards-Silver Prize

Xinxin Chen, University Paris 6

Thesis title: Convergence Rate of the Limit Theorem of a Galton-Watson Tree with Neutral Mutations 
Advisor: Professor Marc Yor

Current affiliation: University Paris 6

Changhao Chen, Hubei University

Thesis title: Factorization of delta-monotone linear mappings

Advisor: Professor Shengyou Wen

Bachelor Thesis Awards-Gold Prize

Mingyi Zhang, Tsinghua University

Thesis title: Topological Classification of simplest Gorenstein non-Complete Intersection Singularities of dimension 2 (Minimally Elliptic singularities with fundamental cycle self intersection -5)

Advisor: Professor Stephen S.-T. Yau

\section{Bachelor Thesis Awards-Silver Prize}

Wei Lu, Tsinghua University

Thesis title: Local and global Euler characteristic formulas

Advisor: Professor Linsheng Yin 